\section{Adapting to the challenge of psychosis: personal resilience and the use of sealing-over (avoidant) coping strategies}

\author{
LYNDA TAIT, MAX BIRCHWOOD and PETER TROWER
}

\author{
Background Avoidance coping (e.g. \\ sealing over) is common in people \\ recovering from psychosis, but it is not \\ understood why some individuals 'seal \\ over'.
}

\begin{abstract}
Aims We examined the hypothesis that individuals who 'seal over' do not have the personal resilience to withstand this major life event.
\end{abstract}

\begin{abstract}
Method Fifty participants were interviewed during an acute episode of psychosis and reassessed at 3-month and 6-month follow-up. Measures included psychotic symptoms, recovery style, service engagement, parental and adult attachment and self-evaluative beliefs.
\end{abstract}

Results Sealing-over recovery styles are associated with negative early childhood experience, insecure adult attachment, negative self-evaluative beliefs and insecure identity. Insecure adult attachment was associated with less engagement with services.

\section{Conclusions Sealing over was associated with multiple signs of low personal resilience in adapting to psychosis.}

\section{Declaration of interest None.} Funded by the School of Psychology, University of Birmingham, UK.
Research investigating recovery in psychosis has shown that people with a diagnosis of schizophrenia often use avoidance coping strategies rather than approach strategies (i.e. 'sealing-over' coping rather than 'integration' coping; McGlashan et al, 1977; McGlashan, 1987; Drayton et al, 1998; Jackson et al, 1998; Tait et al, 2003; Thompson et al, 2003). Recovery style has been identified as an important factor in adjustment to psychosis. Sealing over tends to be associated with poorer social functioning and quality of life and higher levels of depression (McGlashan, 1987; Drayton et al, 1998; Thompson et al, 2003). In contrast to earlier views of recovery style as a stable trait characteristic (e.g. McGlashan, 1987), recent evidence suggests that recovery style can change over time; integration style changing to sealing over during adjustment to psychosis, and vice versa (Jackson et al, 1998; Tait et al, 2003; Thompson et al, 2003). However, it is not yet known why some individuals adopt sealing over as a recovery style rather than an integration recovery style. One promising line of investigation suggests that individuals who seal over are psychologically vulnerable, with little resilience to life change (Drayton et al, 1998). The present study represents an important extension of previous research, which explored the temporal stability of recovery style and its relationship to insight, symptoms and service engagement, by exploring plausible indicators of psychological vulnerability that may underpin or maintain recovery styles. The study was designed to test the following three hypotheses.

(a) Individuals who seal over, compared with those who use integration coping, will evaluate themselves more negatively and perceive their identity as less secure.

(b) Individuals who seal over will describe a history of difficulty with parental and ongoing adult attachment relationships.

(c) Problems of attachment in individuals who seal over will be manifested in poorer engagement.

\section{METHOD}

Our methods have been described in detail elsewhere (Tait et al, 2003) and are summarised here.

Fifty individuals with an ICD-10 (World Health Organization, 1992) chart diagnosis of schizophrenia or related disorders (F20, F22, F23, F25) were recruited from two urban mental health services. The Structured Clinical Interview for the Positive and Negative Syndrome Scale (SCI-PANSS; Kay et al, 1987) was used to assess suitable patients. Participants were excluded if they had a primary diagnosis of substance use disorder, mood disorder or organic mental disorder. Assessments were conducted during acute psychosis and at 3-month and 6-month follow-up.

\section{Instruments}

Positive and Negative Syndrome Scale

Severity of psychosis was measured with the SCI-PANSS (Kay et al, 1987). The 30 -item SCI-PANSS is a widely used, valid and reliable measure for assessing symptoms of schizophrenia.

\section{Recovery Style Questionnaire}

Recovery style was measured with the Recovery Style Questionnaire (RSQ; Drayton et al, 1998). The RSQ is a 39-item selfreport measure, designed to reflect categories consistent with those developed by McGlashan et al (1977). Four recovery styles can be classified: integration; mixed picture in which integration predominates; mixed picture in which sealing over predominates; sealing over. Higher scores represent sealing over. The RSQ was administered at each of the three assessments.

\section{Parental Bonding Instrument}

Recalled parenting behaviours were assessed using the revised version of the Parental Bonding Instrument (PBI; Parker et al, 1997). This 25 -item self-report questionnaire comprises the Protection Scale (13 items) and the Care Scale (12 items). Participants are asked to indicate, on a four-point Likert-style scale, the extent to 
which each item is characteristic of their mother and father. Low scores on the Care Scale reflect perceived parental neglect and rejection, whereas high scores reflect perceived parental warmth and affection. High scores on the Protection Scale indicate perceived excessive control and intrusive parenting, whereas low scores suggest perceived parental acceptance of a child's independence and autonomy. Test-retest reliability values have been reported as 0.77 for the maternal Care Scale, 0.73 for the maternal Protection Scale, 0.58 for the paternal Care Scale and 0.69 for the paternal Protection Scale in a sample of patients diagnosed with schizophrenia (Parker et al, 1982). Perceived parental abuse (physical) was measured with the five-item Measure of Parenting Style (MOPS; Parker et al, 1997). The PBI and MOPS were administered at the 3-month follow-up point, when the individual had recovered from the acute episode. In this study, the Cronbach coefficient $\alpha$ was 0.97 for maternal care, 0.96 for paternal care, 0.67 for maternal protection, 0.76 for paternal protection, 0.94 for maternal abuse and 0.95 for paternal abuse.

\section{Revised Adult Attachment Scale (RAAS)}

Adult attachment style was measured with the revised version of the Adult Attachment Scale (RAAS; Collins, 1996); an adaptation of a self-report measure of adult attachment developed by Collins \& Read (1990). The scale consists of 18 items, 6 on each of three sub-scales: the Close sub-scale refers to the extent to which an individual is comfortable with closeness and intimacy in relationships; the Depend sub-scale measures the degree to which an individual can depend on others; and the Anxiety sub-scale refers to a person's fear of interpersonal rejection. Ratings are made on a five-point scale (1, not at all characteristic of me; 5 , very characteristic of me). An individual with a 'secure' attachment style can tolerate closeness/intimacy and dependence on others and has low anxiety about interpersonal rejection (Collins, 1996). The RAAS was administered at the 3 -month assessment. The Cronbach coefficient $\alpha$ was 0.86 for the Close sub-scale, 0.86 for the Depend sub-scale and 0.97 for the Anxiety sub-scale.

\section{Evaluative Beliefs Scale}

The Evaluative Beliefs Scale (EBS; Chadwick et al, 1999) was used to assess self-evaluative beliefs about self and others. Evaluative beliefs were assessed across three specific dimensions: six items constitute a self-evaluation sub-scale (Self-Self); six items constitute evaluations of other people (Self-Other); and six items constitute a person's beliefs about how other people evaluate them (Other-Self). Higher scores reflect greater negative evaluation. Reliability is reported as excellent (Chadwick et al, 1999), with $\alpha$ for the Self-Self, Other-Self and Self-Other scales reported to be $0.90,0.92$, and 0.86 , respectively. In this study, $\alpha$ for the Self-Self, Other-Self and other Self-Other scales were 0.89, 0.95 and 0.88 , respectively. The EBS was administered at the 3-month assessment.

\section{Service Engagement Scale}

Service engagement was measured with the Service Engagement Scale (SES; Tait et al, 2002). The SES is a 14-item measure, with higher scores indicating lower engagement. The SES was completed at the 6-month assessment. The Cronbach coefficient $\alpha$ was 0.81 for availability, 0.76 for collaboration, 0.90 for help-seeking and 0.82 for treatment adherence.

\section{Self and Other Scale}

Secure self was measured with the Self and Other Scale (SOS; Dagnan et al, 2002). The SOS is a self-report scale with two subscales, the Insecure Self and the Engulfed Self, each consisting of seven items rated on a five-point scale ranging from 'agree strongly' to 'disagree strongly'. The Cronbach coefficient $\alpha$ for the Insecure Self sub-scale was reported as 0.76 , and 0.78 for the Engulfed Self sub-scale. In this study, $\alpha$ was 0.92 for the Insecure Self sub-scale and 0.82 for the Engulfed Self sub-scale. The SOS was administered at the 6-month assessment.

\section{Calgary Depression Scale for Schizophrenia}

Depression was measured with the Calgary Depression Scale for Schizophrenia (CDSS; Addington et al, 1993). The CDSS is a structured interview measure specifically designed for use in samples of individuals diagnosed with schizophrenia: the CDSS distinguishes between depression and negative symptoms, and is reported to be more straightforward to administer than other widely used depression instruments (Addington et al, 1993).
The CDSS is composed of eight structured questions and one interviewer observation of the entire interview. The higher the total score, the higher the level of depression. It has been shown to possess excellent psychometric properties, including good correlation with other wellestablished measures of depression $(r=0.79-0.87)$, with internal consistency $\alpha$ values of 0.71-0.79 (Addington et al, 1993).

\section{Statistical analysis}

Statistical analysis was performed with the Statistical Package for the Social Sciences for Windows, version 10.0.7. The $\chi^{2}$ test and Fisher's exact test were used to test categorical variables. One-way analysis of variance (ANOVA), with planned comparison tests where appropriate, was used for significance in mean differences between groups. Pearson's correlation analysis was used to examine relationships between continuous variables, and a one-sample $t$-test was used to test mean differences between two groups.

\section{RESULTS}

\section{Sample characteristics}

Of 62 service users identified, 12 declined to participate, leaving 50 eligible participants $(81 \%)$. Complete follow-up data were obtained for 46 participants at the second assessment and for 42 participants at the third assessment ( $16 \%$ attrition). There were no significant differences between completers and non-completers on gender, ethnicity, marital status, education, living situation or treatment location. The sample comprised 19 females $(38 \%)$ and 31 males $(62 \%)$; they were predominantly single $(70 \%)$ and living alone $(58 \%)$. The mean age of the sample was 33.8 years $($ s.d. $=12.0)$ and the mean duration since first onset of psychosis was 7.2 years (s.d.=9.71).

\section{Self-evaluation and recovery style Evaluative beliefs}

As indicated in Table 1, there was a significant difference between recovery style groups in Other-Self beliefs $(F(3,42)=$ $5.26, P<0.01)$; the two sealing-over groups scored significantly higher than the two integration groups $(F(1,42)=15.69, P<0.001)$, indicating a more negative view of self. 
Table I Statistics comparing parental bonding, adult attachment, evaluative beliefs and secure self mean scores across each recovery style group

\begin{tabular}{lccccc}
\hline & Integration & Mixed integration & Mixed sealing & Sealing & Significance \\
\hline PBI (Maternal) & & & & & \\
Care sub-scale & 24.4 & 25.6 & 13.5 & 9.5 & $* * *$ \\
Abuse sub-scale & 4.5 & 3.0 & 5.9 & 10.0 & $* *$ \\
Protection sub-scale & 15.8 & 11.7 & 17.9 & 11.7 & NS \\
PBI (Paternal) & & & & & \\
Care sub-scale & 26.7 & 24.7 & 9.8 & 6.3 & $* * *$ \\
Abuse sub-scale & 2.1 & 2.9 & 8.3 & 9.1 & $* * *$ \\
Protection sub-scale & 9.7 & 13.8 & 15.6 & 13.0 & NS \\
RAAS & & & & & \\
Close sub-scale & 24.7 & 18.7 & 16.2 & 14.5 & $* *$ \\
Depend sub-scale & 20.8 & 17.8 & 11.8 & 11.5 & $* * *$ \\
Anxiety sub-scale & 12.9 & 19.5 & 27.1 & 24.2 & $* * *$ \\
EBS & & & & & \\
Other-Self sub-scale & 6.0 & 5.9 & 14.2 & 13.2 & $* *$ \\
Self-Self sub-scale & 4.0 & 3.7 & 6.2 & 6.2 & NS \\
Self-Other sub-scale & 0.9 & 2.6 & 1.2 & 1.8 & NS \\
SOS & & & & & \\
Insecurity sub-scale & 18.8 & 26.7 & 31.4 & 29.5 & $* *$ \\
Engulfed sub-scale & 17.7 & 19.5 & 15.6 & 16.6 & NS \\
\hline
\end{tabular}

EBS, Evaluative Beliefs Scale; PBI, Parental Bonding Instrument; RAAS, Revised Adult Attachment Scale; SOS, Self and Other Scale.

\section{Secure self and recovery style}

There was a significant overall group effect for the SOS, focusing on the Insecurity subscale $\left(F(3,39)=6.09, P<0.1, \eta^{2}=0.32\right)$; the planned contrast indicated that the two sealing-over groups reported significantly higher scores (less secure) than the two integration groups.

\section{Recovery style and interpersonal attachment}

\section{Parental attachment}

Preliminary correlation analysis at the 3-month assessment of the relationship between the PBI and level of depression was undertaken to determine the potential confounding effect of depression on perceived parental bonding (i.e. to examine the possibility that negative mood might be a source of bias in reported memories of perceived parenting). Correlations between the Care, Protection and Abuse sub-scales and concurrent level of depression were unrelated for mothers $(r=0.10, P=0.54$; $r=0.02, P=0.88 ; r=0.11, P=0.50)$ and for fathers $\quad(r=-0.08, \quad P=0.66 ; \quad r=0.03$, $P=0.86 ; \quad r=-0.14, \quad P=0.42$ ). Similarly, there were no significant correlations between any PBI and PANSS scales during follow-up, with the exception of a correlation between PANSS general psychopathology and maternal overprotection $(r=0.39, P=0.007)$, between PANSS total psychopathology and maternal abuse $(r=0.34, P=0.03)$ and between low paternal care and PANSS positive symptoms $(r=-0.35, P=0.025)$.

The means, ANOVA results and significance levels between the four recovery style groups on the PBI scales are presented in Table 1 . There was a significant overall difference for both the Care and Abuse sub-scale scores for mothers $(\mathrm{F}(3,42)=4.81, P<0.01$, $\left.\eta^{2}=0.62 ; F(3,37)=6.07, P<0.01, \eta^{2}=0.33\right)$. Each of the sealing-over groups rated mothers $(F(1,42)=13.41, \quad P<0.001)$ and fathers $(F(1,37)=38.19, P<0.001)$ as significantly less caring and more abusive $(F(1,42)=8.39$, $P<0.01 ; F(1,37)=17.78, P<0.001$, mothers and fathers, respectively) than did each of the two integration groups. There were no significant differences between the four groups in either maternal protection $(F(3,42)=2.12$, $P=0.11)$ or paternal protection $(F(3,37)=$ $1.06, P=0.38)$. These results were unaffected when controlling for PANSS scales.

\section{Adult attachment}

Table 1 indicates that the four recovery style groups differed significantly on the
RAAS Close $(F(3,42)=3.92, \quad P<0.01$, $\left.\eta^{2}=0.22\right), \quad$ Depend $\quad(F(3,42)=5.04$, $\left.P<0.01, \eta^{2}=0.26\right)$ and (rejection) Anxiety sub-scales $\quad(F(3,42)=5.42, \quad P<0.01$, $\eta^{2}=0.28$ ). Planned comparisons revealed that the two sealing-over groups scored lower on the Close $(F(1,42)=7.43$, $P<0.01)$ and Depend $(F(1,42)=13.51$, $P<0.001)$ sub-scales but higher on the Anxiety sub-scale $\quad(F(1,42)=12.20$, $P<0.001)$ than the integration groups.

There were no correlations between the RAAS and the PANSS, with the exception of a correlation of $r=0.31 \quad(P=0.03)$ between RAAS Anxiety and PANSS positive. The four recovery style groups continued to differ significantly on RAAS Anxiety when PANSS positive was controlled $(F(3,42)=7.1, P<0.001)$.

\section{Relationship between childhood and adult attachment}

Attachment theory argues that attachments in childhood provide the cognitive schema that guide adult relationships; the attachment style and mental models of self and others develop early and tend to endure over time (Bowlby, 1973). This study therefore examined the relationship between the PBI and RAAS (see Table 2).

Strong and significant correlations were observed between the RAAS Depend and Close sub-scales and the Care sub-scale of the PBI for mothers and fathers. In contrast, the RAAS Depend and Close sub-scales were inversely related to the PBI Abuse sub-scale, again for both parents. Rejection anxiety in adult relationships (RAAS Anxiety) was significantly correlated with PBI abuse and (lack of) care in both parents. The (over) protection scale did not feature as a significant dimension linking early and adult attachment.

\section{Current adult attachment and service engagement}

A one-sample $t$-test conducted on the SES total scores revealed that the RAAS 'insecurely attached' group had significantly higher SES mean scores (mean 23.72, s.d.=10.74) than the 'securely attached' group (mean 10.07, s.d. $=10.20$ ): $t=3.64$, $P<0.001, \eta^{2}=0.31$. These results reveal that having an insecure attachment style is associated with a greater likelihood of disengaging from mental health services staff. 
Table 2 Intercorrelations between childhood (Parental Bonding Instrument, PBI) and current adult attachment (Revised Adult Attachment Scale, RAAS) ratings

\begin{tabular}{|c|c|c|c|c|c|c|}
\hline \multirow[t]{3}{*}{ RAAS } & \multicolumn{6}{|c|}{ PBI scales } \\
\hline & \multicolumn{2}{|c|}{ Care } & \multicolumn{2}{|c|}{ Protection } & \multicolumn{2}{|c|}{ Abuse } \\
\hline & Mother & Father & Mother & Father & Mother & Father \\
\hline Close & $0.62 * *$ & $0.62^{* *}$ & -0.15 & $-0.35^{*}$ & $-0.54 *$ & $-0.3 I^{*}$ \\
\hline Depend & $0.58^{* *}$ & $0.61^{* *}$ & -0.28 & -0.29 & $-0.58 * *$ & $-0.4 I^{* *}$ \\
\hline Anxiety & $-0.57^{* *}$ & $-0.6 I^{* *}$ & 0.21 & 0.22 & $0.54 * *$ & $0.45^{* *}$ \\
\hline
\end{tabular}

$* P<0.05 ; * * P<0.01$.

\section{Recovery style and psychological adjustment}

Recovery style and depression

Contrary to expectations, the ANOVA results revealed no significant betweengroup differences in the level of depression at the 6-month follow-up $(F(3,38)=1.14$, $\left.P>0.05, \eta^{2}=0.08\right)$.

\section{DISCUSSION}

This study builds on our previous work (Drayton et al, 1998), examining the hypothesis that the sealing-integration dimension of coping that we found to predict service engagement (Tait et al, 2003) is embedded in the psychology of adaptation to trauma. Contrary to previously held assumptions, recent evidence suggests that the recovery style of sealing over is not the same as poor insight; sealing is a dimension of coping that is independent of insight or psychosis symptoms (Drayton et al, 1998; Tait et al, 2003). In this study, a clear relationship emerged between sealing and a perception that others see the individual as worthless and a self-perception of an insecure identity. The study replicates our previous finding that individuals who seal over report a history of attachment difficulty with care-givers (Drayton et al, 1998) but, significantly, finds evidence that this is also manifest in current adult relationships. We interpret these findings to suggest that sealing is adopted by individuals whose psychological resources ('resilience') to deal with this potentially traumatic event are impoverished by virtue of an anomalous development trajectory (Birchwood, 2003).

\section{Methodological issues}

The reliance in this study on retrospective accounts of early childhood experience, and the potential recall bias that may have resulted from depressed mood, raises a question about the reliability of these data. However, statistical analysis indicated little or no bias due to an effect of mood on reported memories of perceived parenting; Duggan et al (1998) reported that depressed patients' retrospective reports of parental relationships, as measured by the PBI, were corroborated by their siblings. Although bias cannot be ruled out, these considerations, and the similarity with other findings in the literature, do support their validity: attachment theory argues that attachments in childhood and adolescence provide the cognitive schema that come into play in adult relationships, so we would expect correlations between early (PBI) and adult (RAAS) attachment measures, which was precisely what was observed. From a methodological point of view, however, the predicted link between self-ratings of childhood and adult relationships argues against retrospective bias in the case of the PBI and supports one of the central propositions of attachment theory, that individuals are accessing schema that endure and are common to both. It should be emphasised, however, that the link between parental attachment and adult functioning in this group does not imply that the arrow of causality runs from parent to child; contemporary child development theory accepts that there may be behavioural anomalies in the child (e.g. arising from a developing psychosis) that some parents may find difficult adapting to, and that these transactions culminate in attachment difficulty (Duggan et al, 1998).

The use of inner-city settings in recruiting participants raises the question that the results may not generalise; for example, there may be a higher rate of parental neglect linked to deprivation in this setting.
However, it should be noted that the participants were, in seven out of ten instances, within 5 years of first onset of psychosis and the experimental sampling method identified patients in acute crisis, including those who relapsed several times. We believe the sample, therefore, to be representative of the trajectories of psychosis in general (Harrison et al, 2001), but we cannot discount the possibility that the level of perceived parental difficulty is inflated.

The single correlation between anxiety about rejection in relationships and PANSS positive symptoms $(r=0.31)$ raises the possibility that this mistrust is partly influenced by psychosis. This did not affect the observed difference between recovery groups, although mistrust in relationships is often observed as a premorbid characteristic and may lie on a continuum with persecutory thinking.

\section{Recovery style and early childhood experience}

This study found that participants with sealing-over recovery styles reported lower levels of parental care during early childhood than those who tended to use integration recovery styles, with no differences in parental protection. These findings are consistent with previous research in which the lack of care dimension appears to be more important to psychological functioning than the protection dimension of the PBI (Mackinnon et al, 1993; Drayton et al, 1998). The results of the present study add further support to the view that anomalies of early childhood experience may have an effect on coping with adversity in adulthood (e.g. Bowlby, 1969; Richman \& Flaherty, 1987).

Previous evidence suggests that other early developmental experiences, such as physically abusive parenting, are important influences on emotional distress in adulthood; for example, Parker et al (1997) reported a link between abusive parenting and an adult diagnosis of depression. The present study adds to this evidence in finding that individuals with sealing-over recovery styles reported higher levels of reported abuse in childhood from both parents compared with individuals with integration recovery styles. However, the level of depression in the present study was unrelated to perceived parenting behaviour. The perception of parenting behaviour was evaluated after controlling for the effect of mood, indicating that the 
PBI scores were independent of the concurrent level of depression. Thus, as indicated above, reported parental behaviour was not the result of response bias related to depression, a finding consistent with previous research (Gerlsma et al, 1994).

\section{Recovery style and adult attachment}

Compared with integration recovery styles, sealing-over recovery styles were associated with participants' reports of more anxiety about interpersonal rejection, as well as with lower levels of comfort with closeness and dependence in relationships. Furthermore, insecurely attached participants were more prone to low engagement with services than were participants who had more secure attachment styles. This finding, that insecure attachment was related to an avoidant style of coping (i.e. sealing over), which in turn predicted less engagement with services, is in line with attachment theory and previous research showing the vulnerability of insecurely attached persons in coping with stressful life experiences (Bowlby, 1969; Mikulincer, 1998). Again, this is in keeping with previous research showing that insecurely attached persons tend towards interpersonal distance (Klohnen \& Bera, 1998) and negative, mistrusting beliefs about other people (Collins \& Read, 1990; Mikulincer, 1998). It seems likely that mental health professionals are also viewed in this way by patients who seal over and have insecure attachment styles, which in turn contribute to lower engagement with services (Adshead, 1998; Tait et al, 2003). In other words, low engagement with psychiatric services and case managers may, in part, reflect attachment concerns. An alternative explanation is that low engagement with psychiatric services is a rational response to maladaptive care strategies adopted by mental health care professionals (Adshead, 1998), or where services are inappropriate or insensitively delivered to clients (Tait $e t$ al, 2002). Indeed, researchers have suggested that the formation of a trusting relationship between mental health care professionals and service users is a task for both parties (Tait et al, 2002).

Contrary to the hypothesis, and the findings of Drayton et al (1998), the results indicate that participants with sealing-over recovery styles do not hold higher levels of negative evaluative beliefs about the self or about others, and are not more depressed, compared with those who use integration recovery styles. However, those who used sealing-over recovery styles also reported higher ratings on the Other-Self sub-scale of the EBS, indicating a vulnerability to believe that others view them in a negative way. This is in line with findings that individuals who seal over also tend to have insecure attachment styles that are represented by concerns about ongoing interpersonal relationships. Although speculative, one possible explanation is consistent with attachment theory (Bowlby, 1969): individuals with sealing-over recovery styles who also have insecure attachment styles may be more likely to misinterpret others' behaviour towards them as rejecting or critical and therefore are more likely to believe that others view them in a negative manner; or alternatively, it is possible that they are more sensitive to actual rejecting behaviour from others and are realistic in their appraisal of others' views about themselves.

\section{Recovery style and view of self}

The results show that individuals who seal over have difficulties with feelings of insecurity and interpersonal rejection; however, they were no more likely than people who integrate to feel a need to protect the self from being controlled by others. This supports the view that a functional sense of self or identity is an important resilience factor in recovery from psychosis, and in facilitating coping efforts (Davidson \& Strauss, 1992); conversely, individuals are unlikely to engage in approach types of coping if they have failed to develop an active and robust identity that goes beyond the confines of the illness (Davidson \& Strauss, 1992).

\section{Therapeutic implications}

The majority of the sample was aged under 30 years and was within 5 years of onset of psychosis. This is a period of high risk of relapse (Robinson et al, 1999) where the 'blueprint' for long-term trajectories is laid down (Harrison et al, 2001). Drug noncompliance is common in this phase and linked to a cycle of relapse (Robinson et al, 1999); without effective service engagement at this stage, our most effective treatments will not have an opportunity to deliver the benefits they promise (Birchwood, 2003). This and our linked study (Tait et al, 2003) together suggest that service engagement is not solely a matter of insight or resolution of psychotic symptoms, but of personal adaptation to the potentially traumatic nature of the diagnosis and its treatment. This analysis proposes two therapeutic avenues.

The first concerns the design of services and the interpersonal behaviour of case managers. Individuals who seal over may be more likely to engage with a service that is 'on tap, but not on top': in other words, one that engages in a low-key, informal way, that keeps the patient in control of the relationship and focuses attention to the broader needs and aspirations of the individual, in a normalising context. Demanding and cajoling compliance with treatment, insensitive use of psychoeducation and stigmatising the individual (e.g. by admission to wards with more chronic patients) would be likely to stoke avoidance and disengagement in this group. Because engagement is a two-way process, additional research to understand the complex effects of the interpersonal relationship between the client and the mental health care professional appears to be of urgent policy importance.

Second, therapeutic attention needs to focus on raising self-esteem through nonintrusive activity; the case manager-client relationship may be seen as a 'test bed' to develop trust in others. Case managers would need to have low expressed emotion in their interpersonal behaviour and to recognise that they may be key figures in restoring the individual's psychosocial development trajectory (Birchwood, 2003). The style of intervention envisaged is the low-key, motivational approach employed in compliance therapy (Kemp et al, 1996).

In conclusion, this study has shown that a sealing-over recovery style is associated with multiple indicators of low personal resilience in adapting to psychosis. Whether sealing over is adaptive in reducing distress during recovery should be explored.

\section{ACKNOWLEDGEMENTS}

Special thanks are given to the service users who participated in this study and we gratefully acknowledge the help and cooperation of the mental health services staff.

\section{REFERENCES}

Addington, D., Addington, J. \& Maticka-Tyndale, E. (1993) Assessing depression in schizophrenia: the 
Calgary depression scale. British Journal of Psychiatry, 163 (suppl. 22), 39-44.

Adshead, G. (1998) Psychiatric staff as attachment figures. Understanding management problems in psychiatric services in the light of attachment theory. British Journal of Psychiatry, 172, 64-69.

Birchwood, M. (2003) Pathways to emotional dysfunction in first-episode psychosis. British Journal of Psychiatry, 182, 373-375.

Bowlby, J. (1969) Attachment and Loss: Vol. I. Attachment. New York: Basic Books.

Bowlby, J. (1973) Attachment and Loss: Vol. 2. Separation. New York: Basic Books.

Chadwick, P., Trower, P. \& Dagnan, P. (1999)

Measuring negative person evaluations: the evaluative

beliefs scale. Cognitive Therapy and Research, 23,

549-559.

Collins, N. L. (1996) Working models of attachment: implications for explanation, emotion, and

behavior. Journal of Personality and Social Psychology, $\mathbf{7}$ 810-832.

Collins, N. L. \& Read, S. J. (1990) Adult attachment, working models, and relationship quality in dating couples. Journal of Personality and Social Psychology, 58 644-663.

Dagnan, D., Trower, P. \& Gilbert, P. (2002) Measuring vulnerability to threats to self-construction: the self and other scale. Psychology and Psychotherapy: Theory, Research and Practice, 75, 279-293.

Davidson, L. \& Strauss, J. S. (1992) Sense of self in recovery from severe mental illness. British journal of Medical Psychology, 65, |3|-|45.

\section{Drayton, M., Birchwood, M. \& Trower, P. (1998)}

Early attachment experience and recovery from psychosis. British journal of Clinical Psychology, 37 269-284.

Duggan, C., Sham, P., Minne, S., et al (1998)

Quality of parenting and vulnerability to depression: results from a family study. Psychological Medicine, 28 |85-19|

Gerlsma, C., Kramer, J. J. A. M., Scholing, B., et al (1994) The influence of mood on memories of parental rearing practices. British Journal of Clinical Psychology, 33, 159-172.

Harrison, G., Hopper, K., Craig, T., et al (200I) Recovery from psychotic illness: a 15- and 25-year international follow-up study. British journal of Psychiatry, 178, 506-517.

Jackson, H., McGorry, P., Edwards, J., et al (1998) Cognitively-oriented psychotherapy for early psychosis (COPE). Preliminary results. British Journal of Psychiatry, 172 (suppl. 33), 93-100.

Kay, S. R., Fiszbein, A. \& Opler, L. A. (1987) The positive and negative syndrome scale (PANSS) for schizophrenia. Schizophrenia Bulletin, 13, 261-276.

Kemp, R., Hayward, P., Applewhaite, G., et al (1996) Compliance therapy in psychotic patients: randomised controlled trial. BMJ, 312, 345-349.

Klohnen, E. C. \& Bera, S. (1998) Behavioral and experiential patterns of avoidantly and securely attached women across adulthood: a 3I-year longitudinal perspective. Journal of Personality and Social Psychology, 74, $211-233$.

Mackinnon, A., Henderson, A. S. \& Andrews, G.

(1993) Parental 'affectionless control' as an antecedent to

\section{CLINICAL IMPLICATIONS}

- Perceived early childhood attachment experience, as well as current adult attachment concerns, may contribute to sealing over the experience of psychosis during clinical recovery.

- A focus on attachment concerns and other psychological vulnerabilities might reduce barriers to collaboration, enhancing engagement with services.

- Intrusive, stigmatising and coercive services are likely to exacerbate sealing and reduce service engagement.

\section{LIMITATIONS}

- The sample size was modest and the results require replication.

- The retrospective accounts of early childhood experience of parenting need to be validated independently.

- Generalisability beyond the inner city is unknown.

LYNDA TAIT, PhD, School of Psychology, University of Birmingham; MAX BIRCHWOOD, DSc, Birmingham Early Intervention Service, Birmingham and Solihull Mental Health Trust and School of Psychology, University of Birmingham; PETER TROWER, PhD, School of Psychology, University of Birmingham, Birmingham, UK

Correspondence: Professor Max Birchwood, School of Psychology, University of Birmingham, Edgbaston, Birmingham BI5 2TT,UK. E-mail: m.j.birchwood.20@bham.ac.uk

(First received 27 November 2003, final revision 27 May 2004, accepted 26 June 2004)

adult depression: a risk factor refined. Psychological Medicine, 23, |35-|4|.

McGlashan, T. H. (1987) Recovery style from mental illness and long-term outcome. Journal of Nervous and Mental Disease, 175, 68I-685.

McGlashan, T. H., Wadeson, H. S., Carpenter,W., et a (1977) Art and recovery style from psychosis. Journal of Nervous and Mental Disease, 164, 182-190.

Mikulincer, M. (1998) Attachment working models and the sense of trust: an exploration of interaction goals and affect regulation. Journal of Personality and Social Psychology, 74, 1209-1224.

Parker, G., Fairley, M., Greenwood, J., et al (1982) Parental representations of schizophrenics and their association with onset and course of schizophrenia. British Journal of Psychiatry, 14I, 573-58I.

Parker, G., Roussos, J., Hadzi-Pavlovic, D., et a (1997) The development of a refined measure of dysfunctional parenting and assessment of its relevance in patients with affective disorders. Psychological Medicine, 27, II93-1203.

Richman, J. A. \& Flaherty, J. A. (1987) Adult psychosocial assets and depressive mood over time: effects of internalised childhood attachments. Journal of Nervous and Mental Disease, 175, 703-712.

Robinson, D., Woerner, M. G., Alvir, J. M. J., et al (1999) Predictors of relapse following response from a first episode of schizophrenia or schizoaffective disorder. Archives of General Psychiatry, 56, 24I-247.

Tait, L., Birchwood, M. \& Trower, P. (2002) A new scale (SES) to measure engagement with community mental health services. Journal of Mental Health, II, 191-198.

Tait, L., Birchwood, M. \& Trower, P. (2003) Predicting engagement with services for psychosis: insight, symptoms and recovery style. British Journal of Psychiatry, 182, 123-128.

Thompson, K. N., McGorry, P. D. \& Harrigan, S. M. (2003) Recovery style and outcome in first-episode psychosis. Schizophrenia Research, 62, 31-36.

World Health Organization (1992) Tenth Revision of The International Statistical Classification of Diseases and Related Health Problems (ICD-10). Geneva: $\mathrm{WHO}$ 